\title{
REFLEXIVITY OF COMMUTATIVE SUBSPACE LATTICES
}

\author{
RICHARD HAYDON
}

(Communicated by Palle E. T. Jorgensen)

\begin{abstract}
A short proof is given of Arveson's reflexivity theorem for strongly closed commutative subspace lattices.
\end{abstract}

When $\mathscr{L}$ is a lattice of commuting selfadjoint projections on a Hilbert space $\mathscr{H}$, one can form the algebra $\operatorname{Alg} \mathscr{L}$ of all $T \in \mathscr{B}(\mathscr{H})$ for which $T P=$ $P T P$ for all $P \in \mathscr{L}$. Given a subalgebra $\mathscr{A}$ of $\mathscr{B}(\mathscr{H})$ one can form the lattice Lat $\mathscr{A}$ consisting of all selfadjoint projections $P$ such that $T P=P T P$ whenever $T \in \mathscr{A}$. It is a theorem of W. B. Arveson [1] that if $\mathscr{L}$ is closed in the strong operator topology, then $\mathscr{L}$ is reflexive, that is to say that $\mathscr{L}=$ Lat $\operatorname{Alg} \mathscr{L}$. In this note we shall give a short proof of this result. Our approach avoids topological measure theory and disintegration of measures, though we do use, in a different guise, the class $\mathbf{A}$ of "pseudo-integral" operators that is the key to Arveson's original proof. Other proofs of the theorem have been given by K. R. Davidson [2] and by V. S. Shul'man [3].

Let $(\Omega, \mathscr{F}, \mu)$ be a finite measure space. We shall write $\mathbf{A}(\mu)$ for the algebra of all linear operators $T: L^{2}(\mu) \rightarrow L^{2}(\mu)$ which are bounded from the $L^{1}$-norm to the $L^{1}$-norm and from the $L^{\infty}$-norm to the $L^{\infty}$-norm. For $T \in \mathbf{A}(\mu)$ we define $\|T\|_{\mathrm{A}}=\max \left\{\left\|T: L^{1} \rightarrow L^{1}\right\|,\left\|T: L^{\infty} \rightarrow L^{\infty}\right\|\right\}$. By interpolation, the norm of $T$ in $\mathscr{B}\left(L^{2}(\mu)\right)$ is less than or equal to $\|T\|_{\mathbf{A}}$, so that the unit ball of $\|\cdot\|_{\mathrm{A}}$ is a subset of the unit ball of $\mathscr{B}\left(L^{2}(\mu)\right)$ for the operator norm. In fact, it a closed subset for the weak operator topology (and hence compact for that topology) since $\|T\|_{\mathbf{A}} \leq 1$ if and only if $(T f \mid g) \leq 1$ whenever the elements $f, g$ of $L^{2}(\mu)$ satisfy $\|f\|_{1} \leq 1$ and $\|g\|_{\infty} \leq 1$ or $\|f\|_{\infty} \leq 1$ and $\|g\|_{1} \leq 1$.

When $\mathscr{L}$ is a sublattice of the $\sigma$-algebra $\mathscr{F}$ the projections $P_{L}: f \mapsto f .1_{L}$ $(L \in \mathscr{L})$ form a lattice in $\mathscr{B}\left(L^{2}(\mu)\right)$. We abuse notation by writing $\operatorname{Alg} \mathscr{L}$ for $\operatorname{Alg}\left\{P_{L}: L \in \mathscr{L}\right\}$.

Theorem. Let $(\Omega, \mathscr{F}, \mu)$ be a finite measure space and let $\mathscr{L}$ be a sublattice of $\mathscr{F}$. For any $F \in \mathscr{F}$

$$
\inf _{L \in \mathscr{L}} \mu(F \triangle L)=\max \left\{\left(T \mathbf{1}_{F} \mid \mathbf{1}_{\Omega \backslash F}\right): T \in \mathbf{A}(\mu) \cap \operatorname{Alg} \mathscr{L},\|T\|_{\mathbf{A}}=1\right\} .
$$

Received by the editors January 29, 1991.

1980 Mathematics Subject Classification (1985 Revision). Primary 47D25. 
Proof. If $L \in \mathscr{L}$ and $T \in \mathbf{A}(\mu) \cap \operatorname{Alg} \mathscr{L}$, then

$$
\begin{aligned}
\left(T \mathbf{1}_{F} \mid \mathbf{1}_{\Omega \backslash F}\right) & \leq\left(T \mathbf{1}_{F \cap L} \mid \mathbf{1}_{\Omega \backslash(L \cup F)}\right)+\left(T \mathbf{1}_{F \cap L} \mid \mathbf{1}_{L \backslash F}\right)+\left(T \mathbf{1}_{F \backslash L} \mid \mathbf{1}_{\Omega \backslash F}\right) \\
& \leq 0+\left\|T: L^{\infty} \rightarrow L^{\infty}\right\| \mu(L \backslash F)+\left\|T: L^{1} \rightarrow L^{1}\right\| \mu(F \backslash L) \\
& \leq\|T\|_{\mathbf{A}} \mu(L \triangle F),
\end{aligned}
$$

so that one inequality $(\geq)$ is easily established. To establish the other, we start with the case where $\mathscr{L}$ is a finite lattice.

Lemma. Let $(\Omega, \mathscr{F}, \mu)$ be a finite measure space and let $\mathscr{L}$ be a finite sublattice of $\mathscr{F}$. For any $F \in \mathscr{F}$ there exist $L \in \mathscr{L}$ and $T \in \operatorname{Alg} \mathscr{L}$ with $\|T\|_{\mathbf{A}}=1$ such that

$$
\mu(F \triangle L)=\left(T \mathbf{1}_{F} \mid \mathbf{1}_{\Omega \backslash F}\right) .
$$

Proof. Let $\mathscr{G}$ be the algebra generated by $\mathscr{L} \cup\{F\}$ and let $A_{1}, \ldots, A_{m}$ be the atoms of $\mathscr{G}$ that are contained in $F, B_{1}, \ldots, B_{n}$ the atoms that are disjoint from $F$. Let $G$ be the set of pairs $(i, j)$ such that there is no $L \in \mathscr{L}$ with $A_{i} \subseteq L, B_{j} \cap L=\varnothing$. If $\mathbf{x}=\left(x_{i, j}\right)_{(i, j) \in G}$ is a family of positive real numbers then we may define an operator $T_{\mathbf{x}}$ in $\operatorname{Alg} \mathscr{L}$ by

$$
T_{\mathbf{x}} f=\sum_{(i, j) \in G} \frac{x_{i, j}}{\mu\left(A_{i}\right) \mu\left(B_{j}\right)}\left(f \mid \mathbf{1}_{A_{i}}\right) \mathbf{1}_{B_{j}} .
$$

We easily calculate the norms

$$
\begin{aligned}
\left\|T_{\mathbf{x}}: L^{1} \rightarrow L^{1}\right\| & =\max _{i} \sum_{j} \frac{x_{i, j}}{\mu\left(A_{i}\right)}, \\
\left\|T_{\mathbf{x}}: L^{\infty} \rightarrow L^{\infty}\right\| & =\max _{j} \sum_{i} \frac{x_{i, j}}{\mu\left(B_{j}\right)}
\end{aligned}
$$

as well as the quantity

$$
\left(T_{\mathbf{x}} \mathbf{1}_{F} \mid \mathbf{1}_{\Omega \backslash F}\right)=\sum_{(i, j) \in G} x_{i, j} .
$$

Let $\delta$ be the maximum of this quantity for $\mathbf{x}$ as above and $\left\|T_{\mathbf{x}}\right\|_{\mathrm{A}} \leq 1$. We shall have proved the lemma if we find an element $L$ of $\mathscr{L}$ with $\mu(F \triangle L) \leq \delta$.

Now $\delta$ is thus the solution of the following linear programming problem: Maximize $\sum_{(i, j) \in G} x_{i, j}$ subject to $x_{i, j} \geq 0, \quad \sum_{j} x_{i, j} \leq \alpha_{i}$ for all $i$, and $\sum_{i} x_{i, j} \leq \beta_{j}$ for all $j$, where $\alpha_{i}=\mu\left(A_{i}\right)$ and $\beta_{j}=\mu\left(B_{j}\right)$. This may be regarded as a network-flow problem: we consider a directed graph whose nodes are $A_{1}, A_{2}, \ldots, A_{m}, B_{1}, B_{2}, \ldots, B_{n}$ together with a "source" $S$ and a "sink" $T$. For each $i$ there is a channel from $S$ to $A_{i}$ with maximum capacity $\alpha_{i}$, for each $j$ there is a channel from $B_{j}$ to $T$ with capacity $\beta_{j}$, and there is a channel of infinite capacity from $A_{i}$ to $B_{j}$ whenever $(i, j) \in G$. Our problem is to find the maximal flow through this network. By the Min-Cut Max-Flow Theorem this maximal flow equals

$$
\min _{C} \sum_{c \in C} \text { capacity of } c \text {, }
$$

where the minimum is taken over sets $C$ of channels such that $S$ is separated from $T$ if all channels in $C$ are removed from the network. Evidently, we 
shall not achieve this minimum if we remove a channel of infinite capacity so that the minimizing $C$ will consist of the channels $S A_{i}$ for $i$ in a certain set $I$, and the $B_{j} T$ for $j$ in some $J$.

We have established the existence of $I$ and $J$ such that

$$
\sum_{i \in I} \alpha_{i}+\sum_{j \in J} \beta_{j}=\delta
$$

and such that for every $(i, j) \in G$ at least one of $i \in I, j \in J$ is true. By the definition of $G$ there exists, whenever $i \notin I$ and $j \notin J$, an element $L_{i, j}$ of $\mathscr{L}$ with $A_{i} \subseteq L_{i, j}$ and $B_{j} \cap L_{i, j}=\varnothing$. Since $\mathscr{L}$ is a lattice the set

$$
L=\bigcap_{j \notin J} \bigcup_{i \notin I} L_{i, j}
$$

is in $\mathscr{L}$. Also $A_{i} \subseteq L$ whenever $i \notin I$ and $B_{j} \cap L=\varnothing$ whenever $j \notin J$, so that

$$
\mu(F \triangle L) \leq \sum_{i \in I} \mu\left(A_{i}\right)+\sum_{j \in J} \mu\left(B_{j}\right)=\delta .
$$

We can now resume the proof of the theorem. Let $\delta=\inf \{\mu(F \triangle L): L \in \mathscr{L}\}$ and let $K$ be the set of all $T \in \mathbf{A}(\mu)$ such that $\|T\|_{\mathbf{A}} \leq 1$ and $\left(T \mathbf{1}_{F} \mid \mathbf{1}_{\Omega \backslash F}\right) \geq \delta$. This set is compact in the weak operator topology. If $\mathscr{L}^{\prime}$ is a finite sublattice of $\mathscr{L}$, then

$$
\begin{aligned}
K \cap \operatorname{Alg} \mathscr{L}^{\prime}=\{T \in K:(T f \mid g) & =0 \text { whenever there exists } L \in \mathscr{L} \\
& \text { such that supp } f \subseteq L \text { and } \operatorname{supp} g \subseteq \Omega \backslash L\}
\end{aligned}
$$

is closed in the weak operator topology, and is nonempty by the lemma. By compactness we deduce that $K \cap \operatorname{Alg} \mathscr{L}$ is nonempty, which is what we wanted to prove.

Corollary. Let $\mathscr{H}$ be a Hilbert space and let $\mathscr{L}$ be a strongly closed lattice of commuting selfadjoint projections on $\mathscr{H}$. Then Lat $\operatorname{Alg} \mathscr{L}=\mathscr{L}$.

Proof. The reduction of the problem on a general Hilbert space to the measuretheoretic version that we have just been looking at is rather standard. Let $Q$ be a selfadjoint projection that is not in $\mathscr{L}$; we have to show that $Q$ is not in Lat $\mathrm{Alg} \mathscr{L}$. Let $\mathscr{M}$ be a maximal abelian selfadjoint subalgebra of $\mathscr{B}(\mathscr{H})$ containing the lattice $\mathscr{L}$; then $\operatorname{Alg} \mathscr{L} \supseteq \mathscr{M}$, so that Lat $\operatorname{Alg} \mathscr{L} \subseteq$ Lat $\mathscr{M}$. It is known that Lat $\mathscr{M} \subset \mathscr{M}$ so that we can certainly assume that $Q \in \mathscr{M}$. Since $\mathscr{L}$ is strongly closed, there exist $f_{1}, \ldots, f_{m} \in \mathscr{H}$ such that

$$
\max _{i \leq m}\left\|(P-Q) f_{i}\right\| \geq 1
$$

for all $P \in \mathscr{L}$. Let $\mathscr{H}_{0}$ be the closed subspace generated by $\mathscr{M}\left\{f_{1}, \ldots, f_{m}\right\}$. The orthogonal projection $P_{0}$ of $\mathscr{H}$ onto $\mathscr{H}_{0}$ is in $\mathscr{M}$ and $\mathscr{M}_{0}=\mathscr{M}_{\mathscr{H}_{0}}$ is a maximal abelian selfadjoint subalgebra of $\mathscr{B}\left(\mathscr{H}_{0}\right)$. We can regard $\mathscr{H}_{0}$ as $L^{2}(\mu)$ and identify $\mathscr{M}_{0}$ with $L^{\infty}(\mu)$ for a suitable finite measure $\mu$. The lattice $\left.\mathscr{L}\right|_{\mathscr{H}_{0}}$ of idempotents in $\mathscr{M}_{0}$ has the form $\left\{P_{L}: L \in \mathscr{L}_{0}\right\}$ for some sublattice $\mathscr{L}_{0}$ of $\mathscr{F}$. The restriction to $\mathscr{H}_{0}$ of $Q$ is $P_{F}$ for some $F \in \mathscr{F}$. The existence in $\mathscr{H}_{0}=L^{2}(\mu)$ of the elements $f_{1}, \ldots, f_{m}$, implies that there is some $\delta>0$ such that $\mu(F \triangle L) \geq \delta$ for all $L \in \mathscr{L}_{0}$. The theorem gives some $T_{0} \in \mathscr{B}\left(\mathscr{H}_{0}\right)$ such that $T_{0} P_{L}=P_{L} T_{0} P_{L}$ for all $L \in \mathscr{L}_{0}$ but $T_{0} P_{F} \neq P_{F} T_{0} P_{F}$. If we define $T \in \mathscr{B}(\mathscr{H})$ by $T=T_{0} P_{0}$, then $T \in \operatorname{Alg} \mathscr{L}$ but $T Q \neq Q T Q$. 


\section{REFERENCES}

1. W. B. Arveson, Operator algebras and invariant subspaces, Ann. of Math. (2) 100 (1974), 433-532.

2. K. R. Davidson, Commutative subspace lattices, Indiana Univ. Math. J. 27 (1978), 479-490.

3. V. S. Shulman, Projection lattices in Hilbert space, Functional Anal. Appl. 23 (1990), 158-159; translation of Funkcional. Anal. i Priložen. 23 (1989), 86-89.

Brasenose College, Oxford, OX1 4AJ, United Kingdom 